\title{
Desensitization of soluble guanylyl cyclase, the NO-receptor, by S-nitrosylation
}

\author{
Nazish Sayed*1, Padmamalini Baskaran1, Focco van den Akker ${ }^{2}$ and \\ Annie Beuve ${ }^{1}$
}

\author{
Address: ${ }^{1}$ Department of Pharmacology \& Physiology, New Jersey Medical School, UMDNJ, Newark, NJ 07103 USA and ${ }^{2}$ Department of \\ Biochemistry, Case Western Reserve University, Cleveland, OH 44106 USA \\ Email: Nazish Sayed* - sayedns@umdnj.edu \\ * Corresponding author
}

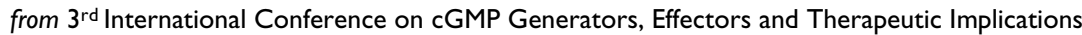

Dresden, Germany. I5-17 June 2007

Published: 25 July 2007

BMC Pharmacology 2007, 7(Suppl I):P50 doi:I0.II86/I47I-22 I0-7-SI-P50

This abstract is available from: http://www.biomedcentral.com/I47I-22I0/7/SI/P50

(c) 2007 Sayed et al; licensee BioMed Central Ltd.

Signaling cascades initiated by nitric oxide (NO) are crucial in the physiology and pathophysiology of the cardiovascular system. NO signal is mediated by the soluble guanylyl cyclase (sGC), which is a heme-containing heterodimer that produces cGMP. In spite of its importance, little is known about the mechanisms of regulation of sGC, even though dysfunctions in the NO-cGMP signaling pathway are thought to be responsible for diseases as prevalent as hypertension and atherosclerosis. In particular, the mechanism of desensitization of sGC remained unresolved. Desensitization of sGC is the loss of sGC activation by NO following an initial exposure to NO.

Post-translational modification has been postulated to affect sGC sensitivity to NO but evidence is still lacking. We show here, that SGC is S-nitrosylated and that this Snitrosylation results in decreased responsiveness to $\mathrm{NO}$ characterized by the loss of NO-stimulated sGC activity. Using primary aortic smooth muscle cells and S-nitrosocysteine (CSNO), we provide evidence that desensitization of sGC is concentration and time dependent, and that sensitivity of sGC to NO is restored, and S-nitrosylation reverses with cellular increase of thiols. We confirmed in vitro using semi-purified sGC and peroxynitrite that S-nitrosylation directly causes desensitization, suggesting that other cellular factors might not be involved. By using Mass Spectrometry, we have identified two cysteines, one in each subunit that are potentially S- nitrosylated. Replacement of these cysteines with Alanine created mutants that were mostly resistant to desensitization. This study suggests that S-nitrosylation of sGC could be a mean by which memory of NO exposure is kept in smooth muscle cells.

\section{Acknowledgements}

We would like to thank Dr. Tong Liu for her exquisite technical expertise in Mass Spectrometry analysis and D. Hong Li as director of the Center for Advanced Proteomics research. This work was supported by $\mathrm{NIH}$ grant GM067640. 